Hantzsch und Kaufmann, Untersalpetrige Säure. 317

$\begin{array}{lcc} & \text { Berechnet für } & \text { Riebensahm: } \\ & \mathrm{C}_{4} \mathrm{H}_{4} \mathrm{BaO}_{6}+\mathrm{H}_{2} \mathrm{O} & \text { Gefunden } \\ \mathrm{H}_{2} \mathrm{O} & 5,9 & 5,8 \\ \mathrm{Ba} & 4 \tilde{5}, 2 & 45,1 \\ & \text { Berechnet für } & \\ & \mathrm{C}_{4} \mathrm{H}_{4} \mathrm{BaO}_{6} & \\ \mathrm{Ba} & 48,1 & 47,9\end{array}$

Mit diesem Salze war das ron Kekulé ${ }^{15}$ ) aus Dibrombernsteinsäure erhaltene Salz höchstwahrscheinlich identisch.

Das mesoweinsaure Baryum ist aber keineswegs in heissem Wasser leicht löslich.

$0,476 \mathrm{~g}$ wurden mit $500 \mathrm{ccm}$ Wasser unter öfterem Umschütteln fünf Stunden lang gekocht, das Ungelöste abfiltrirt und gewogen, die Lösung in einer gewogenen Schale eingedampft, der Verdampfungsrückstand ebenfalls quantitativ bestimmt.

$$
\begin{aligned}
\text { Ungelöst blieben } & 0,174 \mathrm{~g} \\
\text { Der Abdampfrückstand wog } & 0,297 \mathrm{~g} \\
\text { Zusammen } & 0,471 \mathrm{~g} \text { anstatt } 0,476 .
\end{aligned}
$$

Demnach löst sich ein Theil in circa 1600 Theilen kochendem Wasser.

\title{
Zur Kenntniss der untersalpetrigen Säure;
} von A. Hantzsch und Ludwig Kaufmann. (Eingelaufen am 27. Juli 1896.)

Das Studium der von Maumené entdeckten und sodann besonders von Divers, Zorn und van der Plaats untersuchten untersalpetrigen Säure haben wir von zweierlei Gesichtspunkten wieder aufgenommen; erstens wegen der Beziehung dieser Säure zu den eigentlichen Diazoverbindungen, welche durch die Formel HON : NOH und den von W. Wislicenus vorgeschlagenen Namen „Diazosäure" ausgedrückt wird; zweitens

15) Diese Annalen, 1. Suppl., 365 . 
wegen ihrer Beziehungen zu dem von Thiele entdeckten Isomeren, welches von ihm als Nitramid, $\mathrm{NH}_{2} \cdot \mathrm{NO}_{2}$, also als Structurisomeres der bisher noch nicht im freien Zustande bekannten untersalpetrigen Säure angesehen wird.

Wir haben zunächst die Zorn'sche Darstellungsmethode der untersalpetrigen Säure derart verbessert, dass sie stets eine sichere und befriedigende Ausbeute liefert, sodann die freie Säure im festen Zustande gewonnen; desgleichen das bisher noch kaum bekannte Ammoniumsalz und, im Gegensatze zu dem öligen Zorn'schen Aethyläther, den Benzyläther auch in fester Form isolirt. Endlich haben wir Säure, Ester und Salze namentlich auch in physikalisch-chemischer Hinsicht untersucht und soweit als möglich mit dem isomeren "Nitramid" verglichen.

\section{Bildung der Hyponitrite.}

Auf der bis zum Ammoniak reichenden Stufenleiter der Reductionsproducte der Salpetersäure

$$
\mathrm{HNO}_{3}, \mathrm{HNO}_{2},\langle\mathrm{HNO}\rangle_{2}, \mathrm{NH}_{3} \mathrm{O}, \mathrm{NH}_{3}
$$

nimmt die untersalpetrige Säure die Stelle zwischen der salpetrigen Säure und dem Hydroxylamin ein. Daher erscheint ihre Bildung möglich durch Reduction der ihr vorausgehenden oder durch Oxydation der ihr folgenden Glieder.

\section{a) Reductionsmethoden.}

Als Reductionsmittel ist hauptsächlich Natriumamalgam verwendet worden, und zwar entweder direct ${ }^{1}$ ) oder indirect, d. i. bei der Elektrolyse ${ }^{2}$ ) wässriger Natriumnitritlösungen unter Anwendung von Quecksilber als negativem Pol; jedoch konnten die namentlich von Zorn angegebenen Maximalausbeuten von verschiedenen anderen Autoren niemals erhalten werden. Indess hat Tanatar durch Verwendung flüssigen

1) Divers, Chemical News $\mathbf{9 3}, 206$; Zorn, Ber. d. deutsch. chem. Ges. 10, 1306. Hierher gehört anch die von Divers und Haga (Journ. Chem. Soc. 1885, 203-205) ansgeführte interessante Spaltung des sogenannten stickoxydschwefligsauren Kaliums in Hyponitrit und Sulfit.

2) Zorn, Ber. d. deutsch. chem. Ges. 12, 1509 . 
Amalgams eine doppelt so grosse Ausbeute als Divers erzielt. (Ber. d. deutsch. chem. Ges. 26, Ref. 763).

Die Angaben, dass man durch frisch gefälltes Eisenoxydulhydrat ${ }^{3}$ ) oder durch Schmelzen mit Eisenfeile ${ }^{4}$ ) aus Natriumnitrit Hyponitrit erhalten solle, konnte von anderer Seite nicht bestätjgt werden ${ }^{5}$ ). Ebenso wenig erhielten wir durch Reduction von Nitritlösungen mittelst amalgamirten Aluminiums nach dem Verfahren von $\mathrm{H}$. Wislicenus und L. Kaufmann ${ }^{6}$ ) Hyponitrit.

b) Oxydationsmethoden.

Untersalpetrige Säure kann durch Oxydation von Hydroxylamin oder Hydroxylaminderivaten erhalten werden. Die von Thum ${ }^{7}$ ) beobachtete Bildung von untersalpetriger Säure bei der Einwirkung von Hydroxylamin auf einige Metalloxyde, z. B. Quecksilberoxyd, Silberoxyd, Kupferoxyd, beruht auf einer directen Oxydation des Hydroxylamins:

$$
2 \mathrm{NH}_{2} \mathrm{OH}+2 \mathrm{HgO}=2 \mathrm{Hg}+\mathrm{H}_{2} \mathrm{O}+\mathrm{N}_{2} \mathrm{O}_{2} \mathrm{H}_{2} .
$$

Die gerade nach der letzteren Methode erhaltenen Ausbeuten sind ubrigens durchaus nicht so unbedeutend, wie man nach den Angaben von Thum erwarten sollte. So erhielten wir durch Eintragen von überschüssigem Quecksilberoxyd in eine gut gekühlte, aus $5 \mathrm{~g}$ Hydroxylaminsulfat und überschüssigem Alkali bereitete, alkalische Hydroxylaminlösung nach Abfiltriren des Quecksilbers und Neutralisation mit Salpetersäure durch Silbernitrat eine Fällung von $0,5 \mathrm{~g}$ Silberhyponitrit. Es entspricht dies einer Ausbeute von 10 pC. des Gewichtos des Hydroxylaminsulfates oder von etwa $5 \mathrm{pC}$. der Theorie.

Als eigenartige Oxydation des Hydroxylamins zu untersalpetriger Säure erscheint auch die nach Abschluss unserer Arbeit von Piloty ${ }^{8}$ ) veröffentlichte interessante Spaltung der aus

3) Zorn, Ber. d. deutsch. chem. Ges. 15, 1007, 1258.

4) Mencke, Chemical News $\mathbf{3 7}, 270$.

5) Divers und Haga, Chemical News $\mathbf{4 9}, 45$.

6) Ber. d. deutsch. chem. Ges. 28, 1323.

7) Monatsh. f. Chem. 14, 305.

8) Ber. d. deutsch. chem. Ges. \$9, 1560. 
Benzolsulfochlorid und Hydroxylamin gebildete Benzsulfhydroxamsäure durch Alkali in Benzolsulfinsäure und untersalpetrige Säure gemäss der Gleichung:

$2 \mathrm{C}_{6} \mathrm{H}_{5} \mathrm{SO}_{2} \mathrm{NHOH}+2 \mathrm{KOH}=2 \mathrm{C}_{6} \mathrm{H}_{5} \mathrm{SO}_{2} \mathrm{~K}+\mathrm{N}_{2} \mathrm{O}_{2} \mathrm{H}_{2}+2 \mathrm{H}_{2} \mathrm{O}$.

Dieses Piloty'sche Verfahren zur Darstellung der untersalpetrigen Säure liefert relativ sehr gute Ausbeuten.

Durch Einwirkung von salpetriger Säure auf Hydroxylamin entsteht gleichfalls untersalpetrige Säure.

$$
\mathrm{HON} \mathrm{H}_{9}+\mathrm{O} \mathrm{NOH}=\mathrm{HON}: \mathrm{NOH}+\mathrm{H}_{2} \mathrm{O} \text {, }
$$

welcher Process nach dem Vorgange von W. Wislicenus ${ }^{9}$ ) als Condensation bezeichnet werden könnte ${ }^{10}$ ). Die Ausbeuten sind jedoch sowohl bei der Einwirkung von Natriumnitrit auf Hydroxylaminsulfat (Wislicenus) als auch bei derjenigen von Silbernitrit auf Hydroxylaminchlorhydrat (Kretschmer ${ }^{11}$ )) sehr schlecht und oft wechselnd. Auch hier scheint Tanatar's Vorschrift ${ }^{12}$ ) - Einwirkung bei Anwesenheit gewisser Basen relativ die besten Ausbeuten zu liefern.

Ein von uns ausgeführter Versuch, Stickoxyd an Natrium oder Kalium direct zu addiren durch Einleiten des gut getrockneten Gases in flüssiges Natrium- bezw. Kaliumamalgam führte nicht zu Hyponitrit. Es trat nicht die geringste Reaction ein. Ebenso erfolglos waren die bereits vor Jahren von P. Schatzmann im Züricher Laboratorium angestellten, nicht publicirten Versuche, Stickoxydul mit Kaliumhydroxyd unter Druck beziehungsweise bei erhöhter Temperatur zu Hyponitrit zu vereinigen. Es liess sich nur Nitrit und Ammoniak nachweisen.

\section{Darstellung von Silberhyponitrit.}

Da wir zu unserer Untersuchung eine grössere Menge von Silberhyponitrit, dem Ausgangsmaterial für die unter-

9) Ber. d. deutsch. chem. Ges. 26, 771.

10) Vcrgl. übrigens Piloty, Ber. d. deutsch. chem. Ges. 29, 1564.

11) Dissertation, Erlangen 1895, Seite 9.

12) Ber. d. deutsch. chem. Ges. 26, Ref. 763 . 
salpetrige Säure, benöthigten, da ferner zur Zeit der Ausführung dieser Arbeit die oben erwähnte Methode Piloty's noch nicht publicirt war, die übrigen Verfahren aber nur geringe Ausbeuten lieferten bezw. nicht genau beschrieben waren, so gingen wir daran, die relativ beste Methode, die Reduction des Natriumnitrits mit Natriumamalgam, zu verbessern. Wir stellten durch Versuche fest, dass die Ausbeuten wesentlich erhöht werden erstens durch Einhalten tieferer Temperaturen, zweitens durch einen stetigen grossen Ueberschuss des Natriumnitrits gegenüber dem nascenten Wasserstoff und drittens durch ebensolchen an freiem Alkali, da die untersalpetrige Säure nur in stark alkalischer Lösung beständig ist.

Unter Benutzung dieser Thatsachen geben wir zur Darstellung von Silberhyponitrit folgende Vorschrift:

$\mathrm{Zu}$ einer durch eine Kältemischung gut gekühlten Lösung von $20 \mathrm{~g}$ reinem käuflichen Natriumnitrit und $10 \mathrm{~g}$ Natriumhydroxyd in $200 \mathrm{ccm}$ Wasser lässt man aus einem Scheidetrichter tropfenweise flüssiges, aus $16 \mathrm{~g}$ Natrium und $2500 \mathrm{~g}$ Quecksilber bereitetes Natriumamalgam langsam und unter steter starker Bewegung der Flüssigkeit durch einen mittelst Turbine angetriebenen Rührer fliessen, was etwa $3 / 4$ Stunde in Anspruch nehmen soll. Alsdann wird die das Hyponitrit enthaltende Lösung von dem Quecksilber getrennt und mit verdünnter Salpetersäure bei $0^{0}$ so lange versetzt, bis sie nur noch schwach alkalisch reagirt. Hierauf fügt man zur Entfernung des bei der Reduction stets entstehenden Hydroxylamins Quecksilberoxyd nnter Umrühren und Kühlung so lange hinzu, bis neue Mengen nicht mehr verändert werden, d. i., bis der sich rasch zu Boden setzende Niederschlag die gelbe Farbe des Quecksilberoxyds beibehält. Die auf diese Weise vom Hydroxylamin befreite Lösung wird nun unter Anwendung von Pumpe und Nutsche filtrirt; sie lauft stets klar ab, wenn man vorher auf das Filter eine kleine Menge Quecksilberoxyd als Filtrirschicht aufgetragen hat. Das Filtrat wird durch $\mathrm{Zn}$ satz von Salpetersäure genan neutralisirt und sofort mit Silber- 
nitrat versetzt. Der ausfallende Niederschlag von intensiv gelbem und bereits annähernd reinem Silberhyponitrit wird erst mehrmals auf dem Filter und sodann im Erlenmeyerkolben wiederholt mit heissem Wasser ausgewaschen bezw. decantirt, hierauf wieder in sehr verdünnter auf $0^{0}$ abgekühlter Salpetersäure gelöst, rasch filtrirt und mit Ammoniak gefällt. Durch nochmalige Umfällung, d. i. durch Wiederholung dieses Verfahrens erhält man vollkommen reines Silberhyponitrit. Dasselbe kann durch Erhitzen auf dem Wasscrbade oder nach Waschen mit Alkohol und Aether auch im Exsiccator getrocknet werden.

I.

Angewandte Substanz:

Durch Titration ermitteltes Ag:

Berechnet für

AgNo

$\mathrm{Ag}$

78,26
II.

$0,3308 \mathrm{~g}$ $0,2580 \mathrm{~g}$

Das auf diese Weise erbaltene Salz zeigt alle für das Silberhyponitrit in der Litteratur angegebenen Eigenschaften mit Ausnahme der Angabe von van der Plaats, dass es bei $150^{\circ}$ explodire. Trotz zahlreicher Versuche konnten wir dasselbe niemals zur Explosion bringen. - $\mathrm{Zu}$ bemerken ist noch, dass das Silbersalz im zerstrenten Tageslichte seine reingelbe Farbe beibehält, aber im directen Sonnenlichte nach kurzer Zeit einen gelbgrünen Ton annimmt und im feuchten Zustande oberflächlich sogar tiefschwarz wird.

Die Ausbeute an vollkommen reinem Silberhyponitrit aus $20 \mathrm{~g}$ Natriumnitrit beträgt $2-3 \mathrm{~g}$, also etwa $6--7 \mathrm{pC}$. der Theorie. Es entspricht dies der von Zorn angegebenen Maximalausbeute, welche indessen von Anderen, die nach seinen Angaben arbeiteten, niemals erreicht wurde ${ }^{13}$ ).

Es empfiehlt sich nicht, die Reduction mit grösseren Mengen auf einmal, wohl aber mit mehreren Portionen von je $20 \mathrm{~g}$ Nitrit nach einander vorzunehmen und die Reinigung der ersten Silberfällungen gemeinsam auszuführen. Man ge-

13) Vergl. c. Paal, Ber. d. dentsch. chem. Ges. 26, 1026. 
winnt so mit Leichtigkeit in ein bis zwei Tagen circa $20 \mathrm{~g}$ des reinen Salzes. Wir glauben daher, dass diese Darstellung der untersalpetrigen Säure bezw. des Silberhyponitrites aus Natriumnitrit doch die einfachere und billigere bleiben dürfte, da der sehr glatten Spaltung der Benzsulfhydroxamsäure nach Piloty in Benzsulfinsäure und untersalpetrige Säure doch ihre etwas umständliche und kostspielige Darstellungsmethode gegenubersteht.

\section{Die freie untersalpetrige Säure.}

Die untersalpetrige Säure ist in wässriger Lösung zum ersten Male von van der Plaats ${ }^{14}$ ) dargestellt worden. Auch Thum ${ }^{15}$ ) hat sich mit der Untersuchung derselben beschäftigt. Erst Tanatar hat sie etwa gleichzeitig mit uns, jedoch nur in Form eines anscheinend noch wasserhaltigen Oeles gewonnen. In festem Zustand entsteht sie, wie auch Piloty unmittelbar nach unserer vorläufigen Notiz in den ,Berichten d. deutsch. chem. Ges." publicirte, folgendermassen:

In absolut wasserfreien Aether wird sorfältigst getrockneter Chlorwasserstoff eingeleitet. $\mathrm{Zu}$ dieser ätherischen Salzsäure giebt man unter guter Kühlung vollkommen reines und trocknes Silberhyponitrit nach und nach $\mathrm{zu}$, wobei man thunlichst eine Wasseranziehung des Aethers vermeidet. Man fährt mit dem Zusatz von Hyponitrit so lange fort, bis dieses seine gelbe Farbe behält, also bis kein Chlorsilber mehr gebildet wird und keine freie Salzsäure in der ätherischen Lösung mehr nachzuweisen ist. Diese ätherische Lösung wird durch ein trocknes Filter rasch in eine Krystallisirschale filtrirt, sofort in einen Exsiccator gegeben und vor der Pumpe abgedunstet. Hierbei müssen Feuchtigkeit und saure Dämpfe peinlich ferngehalten werden, weshalb sich in dem Exsiccator Schwefelsäure und ein Schälchen mit festem Kali befinden. Man beschleunigt ferner das Verdampfen des Aethers, indem man über denselben

24) Ber. d. deutseh. ehem Ges. 10, 1507.

15) Wiener Monatsh. 14, 294. 
einen durch Kalilauge geleiteten, dann durch Schwefelsäure und schliesslich durch Phosphorpentoxyd getrockneten Luftstrom streichen lässt. Sehr zweckmässig ist es, die Temperatur durch Eintauchen des Exsiccators in ein Gefäss mit Kältemischung möglichst tief $\mathrm{zu}$ halten.

Nach Abdunstung des Aethers erseheint die freie untersalpetrige Säure in Form weisser Krystallblättchen.

Die untersalpetrige Säure ist sehr explosiv. Vollkommen trocken, zersetzt sie sich meist anscheinend ohne jede äussere Veranlassung. Die Zersetzung wird beschleunigt durch die Gegenwart von sauren Dämpfen, vermindert durch starke Kälte. Schon beim Reiben mit dem Glasstabe tritt Verpuffung ein, bei Berührung mit festem Kalihydrat steigert sich dieselbe bis zur Feuererscheinung. - Die untersalpetrige Säure ist nicht nur äusserst leicht löslich in Wasser, also zerfliesslich, sondern auch leicht löslich in Alkohol, ziemlich leicht in Aether, Chloroform und Benzol, schwer löslich in Ligroïn.

Eine Analyse der Säure wurde vergeblich versucht. Aetherfeucht liessen sich die Krystallblättchen einige Zeit halten; sobald sie aber trocken waren, explodirten sie selbst bei $-6^{0}$ und anscheinend spontan, so z. B. durch die Bewegung der Waage beim Versuche, sie zu analysiren.

Trotzdem ist der Nachweis (wenn auch nur in qualitativer Weise) gelungen, dass die erhaltene Verbindung untersalpetrige Säure ist. Es wurden nämlich die Krystalle auf Thon gebracht, im Augenblicke des Trockenwerdens auf ein Uhrglas abgleiten gelassen und in Eiswasser gelöst. Diese wässrige Lösung ergab auf Zusatz von Silbernitrat das charakteristische Silberhyponitrit. Dieselbe Hyponitritreaction ist übrigens auch in dem bei der Zersetzung der Krystalle erhaltenen wässrigen Rückstande deutlich wahrnehmbar. In dem beim Zerfall der untersalpetrigen Säure gemäss der Gleichung

$$
\text { HON : } \mathrm{NOH}=\mathrm{H}_{2} \mathrm{O}+\mathrm{N}_{2} \mathrm{O}
$$

entstehenden Wasser löst sich ein Theil der noch unzersetzten Säure und wird so vor Zersetzung geschützt. 
In wässriger Lösung ist die untersalpetrige Säure bei weitem beständiger als im freien Zustande. Wenn auch, wie später gezeigt werden wird, die Säure in ihrer wässrigen Lösung schon bei einer Temperatur von $25^{\circ}$ ziemlich rasch zerfällt, so ist sie doch bei $0^{0}$ so lange haltbar, dass ihr Molekulargewicht durch Gefrierpunktserniedrigung bestimmt werden kann.

Molekulargewichtsbestimmung. Es wurde zu diesem Zwecke reines Wasser, dessen Frstarrungspunkt im Beckmann'schen Apparate bestimmt worden war, durch Einleiten von reinem Chlorwasserstoff und entsprechendes Verdünnen in eine $1 / 6 \mathrm{n}$-Salzsäure verwandelt.

$20 \mathrm{ccm}$ dieser, $0,1217 \mathrm{~g} \mathrm{HCl}$ enthaltenden Flüssigkeit hatten im Mittel aus fünf Versuchen eine Depression von $0,625^{\circ}$ bewirkt. Alsdann wurde in dieselbe Lösung, während der Beckmann'sche Apparat sich in einer Kältemischung befand, überschüssiges Silberhyponitrit eingetragen. Die so erhaltene $1 / 6 \mathrm{n}$-untersalpetrige Säure $(0,1035 \mathrm{~g}$ in $20 \mathrm{ccm})$ zeigte bei nunmehriger Gefrierpunktsbestimmung nur noch eine Erniedrigung von $0,167^{\circ}$ (Mittel aus drei Beobachtungen).

Die durch äquivalente Mengen Salzsäure und untersalpetrige Säure hervorgerufenen Gefrierpunktserniedrigungen verhalten sich also wie $1: 4$ (genau wie $1: 3,8$ ). Da nun die Salzsäure bei der eingehaltenen Verdünnung (Molekulargewicht in 6000 Litern) völlig dissociirt ist und dementsprechend auch bei unseren Versuchen das Molekulargewicht 18,4 d. i. die Jonenzahl $=2$ ergiebt, so muss bei der Verwandlung von $2 \mathrm{HCl}=$ $(2 \mathrm{H}+2 \mathrm{Cl})$ in $\mathrm{H}_{2} \mathrm{~N}_{2} \mathrm{O}_{2}$ die $\mathrm{Zahl}$ der Molekeln auf den vierten Theil sinken; dies ist nur möglich, wenn die untersalpetrige Säure selbst bei dieser grossen Verdünnung (Molekulargewicht in 3000 Litern) nicht merklich in Jonen gespalten, also eine sebr schwache Säure ist, was durch die später zu besprechenden Leitfähigkeitsbestimmungen in der That bestätigt wurde. Daraus ergiebt sich:

Annalen der Chemie 292. Bd. 
Molekulargewicht:

$$
\begin{gathered}
\text { Berechnet für } \\
\mathrm{H}_{2} \mathrm{~N}_{2} \mathrm{O}_{2}
\end{gathered}
$$

62
Gefunden

Damit ist zugleich ein directer Beweis geliefert, dass die untersalpetrige Säure unter diesen Bedingungen nicht als einfaches Molekül

auftritt.

$$
\mathrm{N} \mathbb{H}_{\mathrm{H}}^{\mathrm{O}}
$$

Verhalten gegen Alkalien. Erinnert schon hiernach die untersalpetrige Säure durch ihre jedenfalls sehr minimale Dissociation an die Kohlensäure, so geschieht dies in noch auffälligerer Weise durch ihr Verhalten bei der Titration unter Anwendung von Phenolphtaleïn als Indicator. Wie früher Thum ${ }^{16}$ ) schon gezeigt hat, wird die freie untersalpetrige Säure hierbei nur zur Hälfte titrirt. Diese Angabe wird durch folgenden Versuch bestätigt :

In $20 \mathrm{ccm} 1 / 10 \mathrm{n}$-Salzsäure wurde überschüssiges Silberhyponitrit eingetragen; die entstandene untersalpetrige Säure wurde bei $0^{0}$ sofort mit Barytwasser unter Anwendung von Phenolphtaleïn bis zur Röthung titrirt.

$$
\begin{array}{r}
\text { Berechnet für die Bildung von } \mathrm{BaN}_{2} \mathrm{O}_{2}: 10,9 \mathrm{ccm} \\
\text { Berechnet für die Bildung von baHN } \mathrm{O}_{2}: 5,45 \mathrm{ccm} \\
\text { Gefunden: } 5,50 \mathrm{ccm} .
\end{array}
$$

Das bei der Titration entstehende Mononatriumhyponitrit reagirt also wie Mononatriumcarbonat neutral auf Phenolphtalein, so dass hierin eine Analogie besteht zwischen<smiles>[W]O[W]</smiles>

Das saure Hyponitrit ist übrigens in der wässrigen Lösung nicht beständig. Wenn man nämlich die Rothfärbung der bis auf den neutralen Punkt titrirten Lösung durch einen Tropfen Salzsäure zum Verschwinden bringt und nun die entfärbte

16) Wiener Monatsh. 14, 294. 
Lösung bei gewöhnlicher Temperatur stehen lässt, so tritt nach kurzer Zeit wiederum die Rothfärbung auf; das primäre Natriumhyponitrit zerfällt also langsam in Aetznatron und Stickoxydul gemäss der Gleichung:

$$
\mathrm{N}_{2} \succ_{\mathrm{OH}}^{\mathrm{ONa}}=\mathrm{N}_{2} \mathrm{O}+\mathrm{NaOH}
$$

Diese Zersetzung lässt sich zeitlich verfolgen. So wurde z. B. in einem Falle durch Zunahme des Alkalititers bestimmt, dass sich bei Zimmertemperatur nach 18 Stunden 43 pC. der ursprünglich als saures Hyponitrit vorhandenen Säuremenge zersetzt hatten.

Reactionen der untersalpetrigen Süure. Entgegen den Angaben von van der Plaats scheiden nach Thum ${ }^{17}$ ) reine Lösungen der freien untersalpetrigen Säure aus Jodkalium kein Jod ab. Auch wir fanden in Uebereinstimmung mit Thum, dass beim Zusammengeben einer reinen, frisch bereiteten Lösung der freien Säure und einer mit Essigsäure versetzten Jodkaliumstärkelösung im ersten Augenblicke keine oder nur eine äusserst geringe Färbung auftritt; diese Färbung wird aber nach einiger Zeit intensiver und tritt ebenso in den längere Zeit gestandenen rein wässrigen Lösungen der freien Säure sofort auf, wodurch sich die entgegengesetzten Angaben von van der Plaats erklären.

Noch bemerkenswerther ist, dass man nach Eintragen des Silberhyponitrits in gut gekiihlte concentrirte Schwefelsäure mit Diphenylamin eine äusserst intensive Blaufärbung und durch Ueberschichten mit Eisensulfat den bekannten braunen Ring erhält, also lauter Reactionen, welche der salpetrigen Säure bezw. der Salpetersäure zukommen. Diese Zersetzungen werden in dem später folgenden Abschnitte über den Zerfall der untersalpetrigen Säure näher behandelt und erklärt werden.

17) Wiener Monatsh. 14, 294. 


\section{Ammoniumhyponitrit,} $\mathrm{H}_{4} \mathrm{~N} . \mathrm{O} . \mathrm{N}: \mathrm{N} . \mathrm{OH}$.

Dieses Salz war bisher noch nicht bekannt. Zorn beobachtete, dass die aus Chlorammoniumlösung und Silberhyponitrit dargestellte Lösung, welche Ammoniumhyponitrit enthalten musste, sich bereits bei gewöhnlicher Temperatur unter Gasentwickelung zersetzte. Die Isolirung eines Ammoniumsalzes gelingt jedoch, wenn man in die nach obiger Angabe bereitete absolut ätherische Lösung der freien Säure gut getrocknetes Ammoniak unter Kühlung einleitet. Nach kurzer Zeit fällt das weisse Ammonsalz aus, das rasch abfiltrirt, mit Aether nachgewaschen und auf Thon getrocknet wird.

Ammoniumhyponitrit schmilzt bei $64-65^{\circ}$ unter stürmischer Zersetzung. Es ist in Wasser leicht und mit alkalischer Reaction löslich, konnte aber aus dieser wie auch aus der alkoholischen Lösung selbst durch Abdunsten im Vacuum nicht wiedergewonnen werden. Diese Flüssigkeiten hinterliessen keinen Rückstand. Die Angabe Jackson's ${ }^{18}$ ), dass durch Eintragen von Silberhyponitrit in alkoholisches Ammoniumsulfid und Abdunsten des Alkohols, das Ammoniumbyponitrit in Form schöner Krystalle erhalten werde, muss deshalb auf einen Irrthume beruhen. - Das feste Salz zersetzt sich schon bei gewöhnlicher Temperatur langsam in Ammoniak, Wasser und Stickoxydul und ist daher nicht zur Gewichtsconstanz zu bringen.

Deshalb war auch von der Analyse kein sehr genaues Resultat zu erwarten. Die Bestimmung des Ammoniaks geschah durch Zersetzung des frisch bereiteten und sofort (ohne Rücksicht auf Gewichtsabnahme) möglichst rasch gewogenen Salzes mit Kalilauge, Uebertreiben des Ammoniaks in $n$-Schwefelsäure und Zurücktitration der letzteren. Drei nach einander mit Proben ein und desselben Präparates ausgeführte Bestimmungen bewiesen indess unzweideutig die Formel des sauren Salzes.

18) Ber. d. deutsch. chem Ges. 27, Ref. 562. 
I. $0,0560 \mathrm{~g}$ Ammoniumsalz : 20,34 pC. $\mathrm{NH}_{3}$.

$\begin{array}{rll}\text { II. } 0,0947 \mathrm{~g} & , & : 20,10 \mathrm{pC} \cdot \mathrm{NH}_{3} \text {. } \\ \text { III. } 0,0631 \mathrm{~g} & , & : 19,94 \mathrm{pC} \cdot \mathrm{NH}_{3} \text {. }\end{array}$

Für $\mathrm{NH}_{4} \mathrm{HN}_{2} \mathrm{O}_{2}$ berechnet: $21,52 \mathrm{pC}$.

Für $\left(\mathrm{NH}_{4}\right)_{2} \mathrm{~N}_{2} \mathrm{O}_{2} \quad, \quad 35,42 \mathrm{pC}$.

Eine Stickoxydulbestimmung durch Zersetzung mit concentrirter Schwefelsäure im Eudiometerrohre wurde nicht mehr versucht, nachdem bei dem ersten Versuche eine so heftige Explosion eingetreten war, dass das Rohr zertrümmert wurde.

Die freiwillige Zersetzung des Ammoniumsalzes wurde auch durch Verfolgung der Gewichtsabnahme im Exsiccator genauer studirt. Nachfolgende Zahlen zeigen, dass das Salz sehr. rasch zerfällt.

\begin{tabular}{|c|c|c|c|}
\hline \multicolumn{2}{|c|}{ Zeit } & Gewicht & $\begin{array}{c}\text { Gewichtsabnahme } \\
\text { in Procenten }\end{array}$ \\
\hline & 0 & $0,1057 \mathrm{~g}$ & - \\
\hline Nach & $87^{\mathrm{h}}$ & $0,0214 \mathrm{~g}$ & 79,75 \\
\hline$n$ & $119^{\mathrm{h}}$ & $0,0169 \mathrm{~g}$ & 84,0 \\
\hline & $167^{\mathrm{h}}$ & $0,0136 \mathrm{~g}$ & 87,13 \\
\hline
\end{tabular}

Diese Zersetzung des sauren Ammoniumhyponitrits erinnert an die des Ammoniumbicarbonates, nur dass letzteres doch bedeutend beständiger ist. - Neutrales Ammoniumbyponitrit, $\left(\mathrm{NH}_{4}\right)_{2} \mathrm{~N}_{2} \mathrm{O}_{2}$, konnte auch durch anhaltendes Einleiten von Ammoniak in die Lösung der untersalpetrigen Säure niemals erhalten werden. Die Analogie mit der Kohlensäure ist auch hier wiederum beachtenswerth; denn neutrales Ammoniumcarbonat geht bekanntlich ziemlich rasch an der Luft in saures Carbonat über.

\section{Benzylhyponitrit, $\mathrm{C}_{7} \mathrm{H}_{7} . \mathrm{O} . \mathrm{N}: \mathrm{N} . \mathrm{OC}_{7} \mathrm{H}_{7}$.}

Der einzige bisher bekannte Ester der untersalpetrigen Säure, das Aethylhyponitrit, wurde bekanntlich von $Z$ orn aus Jodäthyl und Silberhyponitrit in Gestalt eines gelben Oeles erhalten, dessen Dampfdichte trotz seiner Explosibilität bestimmt warde und zur Formel $\left(\mathrm{C}_{2} \mathrm{H}_{5}\right)_{2} \mathrm{~N}_{2} \mathrm{O}_{2}$ führte.

Viel beständiger ist der schön krystallisirende, von uns dargestellte Benzylester. 
Man trägt unter guter Kühlung reines trocknes Silberlyponitrit im Ueberschuss in eine ätherische Lösung von sorgfältig gereinigtem Benzyljodid ein und kühlt bei Eintritt einer stärkeren Erwärmung. Wenn nach etwa zwei bis drei Stunden in einer herausgenommenen Probe der ätherischen Lösung der Geruch nach Benzyljodid nicht mehr wahrnehmbar ist, verdunstet man das ätherische Filtrat und erbält den reinen Benzylester durch Umkrystallisiren aus leichtflüchtigem Ligroïn in schönen Blättern.

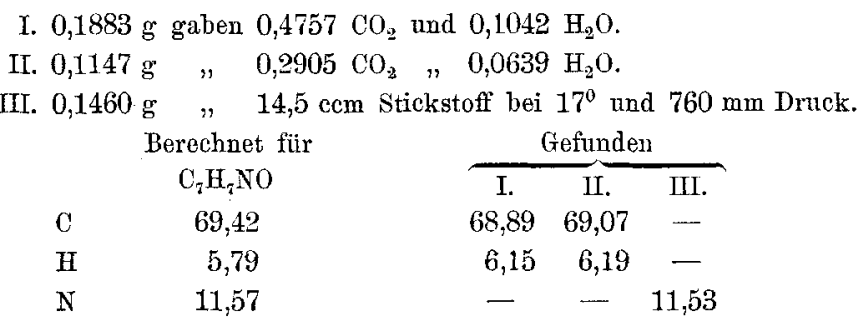

Das Molekulargewicht des Esters wurde in eisessigsaurer Lösung kryoskopisch bestimmt, wobei über die Flüssigkeit gemäss den Angaben von Beckmann ein constanter trockner Luftstrom geleitet wurde.

I. $0,1041 \mathrm{~g}$, in $20 \mathrm{ccm}$ Eisessig (spec. Gew. 1,05̃5) gelöst, erniedrigten dessen Gefrierpunkt um $0,081^{0}$ (Mittel aus sechs Beobachtungen).

II. $0,3086 \mathrm{~g}$, in $20 \mathrm{ccm}$ Eisessig gelöst, erniedrigten den Gefrierpunkt um 0,251 (Mittel aus sieben Beobachtungen).

Molekulargewicht:

Berechnet für $\mathrm{C}_{14} \mathrm{H}_{14} \mathrm{~N}_{2} \mathrm{O}_{2}$

242

$\overbrace{\begin{array}{c}\text { I. } \\ 236\end{array}}^{\text {II. } 227}$

Das Benzylhyponitrit ist also wie die freie Säure und der Zorn'sche Aethylester bimolekular. Es ist in Alkohol und Aether leicht löslich, in Ligroïn schwer löslich und daher aus seiner ätherischen Lösung durch Ligroïn fällbar. Es schmilzt bei $43-45^{\circ}$ unter Zersetzung, explodirt bei raschem Erhitzen auf $60^{\circ}$ und verpufft auch beim Reiben. Schon bei gewöhnlicher Temperatur ist der Ester merklich flüchtig; in 
einem bestimmten Falle konnte eine Gewichtsabnahme von $7,5 \mathrm{pC}$. innerhalb 17 Stunden constatirt werden.

Die Zersetzung des Esters beim Erhitzen findet unter lebhafter Gasentwickelung statt. Zur Untersuchung der Natur des entweichenden Gases wurde eine abgewogene Menge des Benzylhyponitrites in Methylalkohol gelöst und das beim Erwärmen im Kohlensäurestrome auf $50^{\circ}$ aus dieser Lösung entwickelte Gas in einem Stickstoffmesser aufgefangen.

$0,1915 \mathrm{~g}$ gaben $17,7 \mathrm{ccm}$ Gas (bei $18^{\circ}$ und $751 \mathrm{~mm}$ Druck) und zwar Stickstoff, nicht Stickoxydul; danach:

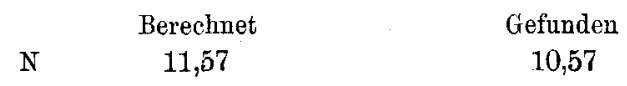

Da der Stickstoff sich also nahezu quantitativ abspaltet, erfolgt wohl die Spaltung zweifellos im Sinne folgender Gleichung:

$\mathrm{C}_{6} \mathrm{H}_{6} \cdot \mathrm{CH}_{2} \mathrm{ON}: \mathrm{NOCH}_{2} \mathrm{C}_{6} \mathrm{H}_{5}=\mathrm{C}_{6} \mathrm{H}_{5} \mathrm{CH}_{2} \mathrm{OH}+\mathrm{C}_{6} \mathrm{H}_{5} \mathrm{CHO}+\mathrm{N}_{2}$.

Hierdurch werden die ohne quantitative Belege angeführten Angaben Zorn's über die analoge Spaltung des Aethylesters bestätigt.

\section{Zerfall der untersalpetrigen Säure.}

Bisher scheint man allgemein angenommen zu haben, dass die untersalpetrige Säure glatt in Stickoxydul und Wasser zerfalle. Die bereits oben angeführte allmähliche Bläuung angesäuerter Jodkaliumstärkelösungen gegenüber der Indifferenz der frischbereiteten wässrigen Säure, ferner die in schwefelsaurer Lösung erfolgende Bräunung von Ferrosulfat und Bläuung von Diphenylamin, endlich auch die später zu beschreibenden Beobachtungen bei den Leitfähigkeitsbestimmungen sind indess kaum anders zu deuten, als dass in Folge einer Nebenreaction aus untersalpetriger Säure auch salpetrige Säure entsteht.

Die nächstliegende Vermuthung war wohl die, dass die untersalpetrige Säure unter gewissen Bedingungen partiell im umgekehrten Sinne ihrer Synthese nach W. Wislicenus gemäss der Gleichung

$$
\mathrm{HON}: \mathrm{NOH}+\mathrm{H}_{2} \mathrm{O}=\mathrm{HONO}+\mathrm{NH}_{2} \mathrm{OH}
$$


in salpetrige Säure und Hydroxylamin gespalten werde. Indess so leicht sich das erstere Spaltstück nachweisen liess, so wenig konnte das letztere selbst unter den zu seiner Erhaltung günstigsten Bedingungen auch nur indirect nachgewiesen werden.

Dagegen ist festgestellt worden: die untersalpetrige Säure zerfällt theilweise nach der Gleichung

$$
3 \mathrm{~N}_{2} \mathrm{O}_{2} \mathrm{H}_{2}=2 \mathrm{~N}_{2} \mathrm{O}_{3}+2 \mathrm{NH}_{3}
$$

in salpetrige Säure und Ammoniak.

Diese eigenthümliche Thatsache lässt sich am besten durch die Annahme erklären, dass die Säure HON : NOH zunächst in ihre tautomere Nebenform von halber Molekulargrösse $0:$ N.H, deren Ester im Nitrosobenzol vorliegt, übergeht; dass dieser Aldehyd der salpetrigen Säure sich indess spontan (ähnlich der Wirkung der echten Aldehyde) auf Kosten eines zu Ammoniak reducirten Moleküls zur Säure oxydirt:

$$
\begin{aligned}
\mathrm{HON}: \mathrm{NOH} & =2 \mathrm{HNO} ; \\
3 \mathrm{HNO} & =\mathrm{NH}_{3}+\mathrm{N}_{2} \mathrm{O}_{3} .
\end{aligned}
$$

Secundär kann ferner das eine Molekül Ammoniak durch die Hälfte der gebildeten salpetrigen Säure oxydirt werden, so dass nach der Gleichung .

$$
\mathrm{NH}_{3}+\mathrm{N}_{3} \mathrm{O}_{3}=\mathrm{N}_{2}+\mathrm{H}_{2} \mathrm{O}+\mathrm{HNO}_{2}
$$

zuletzt Stickstoff und salpetrige Säure bezw. durch deren Oxydation Salpetersäure übrig bleibt.

Diese Spaltungen erfolgen indessen gegenüber dem Zerfall in Stickoxydul und Wasser in rein wässriger Lösung nur langsam. Eine aus überschüssigem Silberhyponitrit und Salzsäure frisch bereitete Lösung wurde sogar durch sofortiges starkes Kochen am Rückflusskühler völlig neutral und gab dementsprechend keine Nitritreaction.

Wurde eine ebenso dargestellte Lösung von bekanntem Gehalt aber erst mehrere Stunden bei $25^{\circ}$ stehen gelassen und dann am Rückflusskühler anhaltend gekocht, so behielt sie ihre saure Reaction bei, enthielt zufolge einer Titration sogar noch etwa 2 pC. Säure und gab neutralisirt und zur Trockne verdampft die Reaction mit Diphenylamin sehr deutlich. - Eine 
zweite gleichbehandelte Probe wurde nach dem Kochen mit Salzsäure eingedampft; im Rückstande war mit Nessler'schem Reagens deutlich Ammoniak nachweisbar. Ferner wurde eine frischbereitete Säurelösung in drei Theile getheilt, der erste intact gelassen, der zweite mit überschüssiger Kalilauge, der dritte mit einigen Tropfen Salzsäure versetzt und diese Lösungen nach 24 Stunden geprüft. Im ersten Theile, der ursprünglich nur untersalpetrige Säure enthielt und deshalb anfänglich keine Jodausscheidung zeigte, war nach 24 Stunden eine intensive Salpetrigsäurereaction, keine Ammoniakreaction und die Gegenwart von noch unveränderter untersalpetriger Säure zu constatiren. Im zweiten, alkalischen Theile war weder salpetrige Säure, noch Ammoniak, sondern nur die noch unverändert erhaltene untersalpetrige Säure nachweisbar. In der freie Salzsäure enthaltenden dritten Partie war weder salpetrige Säure, noch Ammoniak, noch untersalpetrige Säure, sondern ausschliesslich Salpetersäure (durch die Reaction mit Eisensulfat und mit Diphenylaminreaction) vorhanden. Diese drei Versuche zeigen gleichzeitig wiederum: Die untersalpetrige Säure ist am beständigsten in alkalischer, am unbeständigsten in saurer Lösung; die Beständigkeit der rein wässrigen Lösung der freien Säure steht in der Mitte zwischen den beiden Extremen. Dementsprechend war in der alkalischen Lösung keine salpetrige Säure, sondern nur unveränderte untersalpetrige Säure, in der sauren Lösung dagegen keine untersalpetrige Säure mebr, sondern Salpetersäure (durch Oxydation der salpetrigen Säure entstanden) und in der rein wässrigen Lösung beide Säuren nachweisbar.

Dass Ammoniak bei den drei letzterwähnten Versuchen nicht aufgefunden wurde, ist nicht zu verwundern. Dasselbe wird bei dieser langsam und höchstens in schwach saurer Lösung verlaufenden Spaltung durch die gleichzeitig auftretende salpetrige Säure zerstört; es scheint sich also nur durch rasches Eindampfen stark saurer Lösungen im Rückstande nachweisen zu lassen. 
Die Entstehung von salpetriger Säure aus untersalpetriger Säure erklärt nunmehr auch die früher angegebene Reaction der Säure bezw. des Silbersalzes gegen Diphenylamin und Ferrosulfat, namentlich aber auch das erst allmähliche Eintreten der Bläuung einer angesänerten Jodkaliumstärkelösung.

Dieselbe Reaction erklärt es auch, dass, nach den folgenden Versuchen, die Zerfallsgeschwindigkeit der untersalpetrigen Säure in Stickoxydul und Wasser nicht bestimmt werden konnte.

Vor Kenntniss dieser Nebenreaction war zu erwarten, dass das Verhältniss zwischen noch unveränderter Säure (a) und (zu Wasser und Stickoxydul) zersetzter Säure (x) in jedem Zeitmoment durch Titration bezw. Rückgang des Titers zu bestimmen wäre. Daraus hätte sich nach der Gleichung der monomolekularen Processe

$$
c=\frac{1}{t} \cdot \log \frac{a}{a-x}
$$

eine constante Zersetzungsgeschwindigkeit èrgeben müssen. So wurden bei verschiedenen Versuchen einer zuerst bei $0^{0}$ bereiteten und dann im 0 stwald'schen Thermostaten auf $25^{\circ}$ gehaltenen Säurelösung von bekanntem Gehalt zu bestimmten Zeiten je $10 \mathrm{ccm}$ entnommen und mit $1 / 16$ n-Barythydrat unter Anwendung von Phenolphtaleïn als Indicator titrirt. Allein die nach der obigen Gleichung berechneten Werthe für $\mathbf{c}$ waren nicht annähernd constant, sondern nahmen mit zunehmender Zeit so stark $\mathrm{zu}$, dass auf ihre Wiedergabe verzichtet wird.

Die allmähliche Zunahme der Werthe der Zersetzungsgeschwindigkeit mit der Zeit dürfte also durch die allmählich eintretende Nebenreaction bezw. durch die nach ihr auftretende salpetrige Säure veranlasst sein, also ein neues Beispiel von der katalytischen bezw. beschleunigenden Wirkung der Wasserstoffionen darstellen. Denn auch in diesem Falle liess sich die Bildung von salpetriger Säure, bezw. bei Luftzutritt von Salpetersäure, nachweisen. 
Dass Säuren die Zersetzung der untersalpetrigen Säure ungemein beschleunigen, wurde in einem besonderen Versuche durch Bestimmung der Zerfallsgeschwindigkeit bei Anwesenheit von Salzsäure nachgewiesen. Die Anfangswerthe von c waren alsdann sehr viel grösser als die in rein wässriger Lösung. Aber auch in diesem Falle lässt sich aus der Titerabnahme keine Constante berechnen. Trägt man aber die für die Grösse

$$
c=\frac{1}{t} \cdot \log \frac{a}{a-x}
$$

berechneten Werthe als Abscissen, die zugehörigen Zeiten als Ordinaten in ein rechtwinkliges Coordinatensystem ein, so erhält man eine stetig verlaufende Curve, die einer die $x$ - und $y$-Axe schneidenden Geraden sehr nahe kommt. Eine solche Curve besagt, dass die Geschwindigkeit des Zerfalls proportional der Menge des zerfallenden Körpers abnimmt.

Die Ursache dieser eigenthümlichen Erscheinung aufzuklären, bleibt weiteren Versuchen vorbehalten.

\section{Leitfähigkeit der untersalpetrigen Säure.}

Ein durch mehrmaliges Umfällen gereinigtes Silberhyponitrit wurde im Ueberschuss in eine $1 / 32 \mathrm{n}$-Salzsäure bei $0^{0}$ eingetragen. Die hierdurch entstandene $1 / 32$ n-Lösung von untersalpetriger Säure wurde bei $0^{0}$ filtrirt und das durch Spuren von Chlorsilber getrübte, aber keine Salzsäurereaction mehr zeigende Filtrat direct zu den Leitfähigkeitsbestimmungen verwendet.

I. Bestimmung. Gramm-Molekulargewicht in 64 Litern; Temperatur $0^{0}$.

Zufolge zwölf direct aufeinanderfolgenden Einstellungen erwies sich die molekulare Leitfähigkeit $\mu$ sehr gering, wuchs aber mit der Zeit sehr merklich.

$$
\begin{array}{lll}
\mu_{1}=2,98 & \mu_{5}=3,65 & \mu_{9}=4,14 \\
\mu_{2}=3,06 & \mu_{6}=3,78 & \mu_{10}=4,24 \\
\mu_{3}=3,40 & \mu_{7}=3,87 & \mu_{11}=4,29 \\
\mu_{4}=3,46 & \mu_{3}=3,92 & \mu_{12}=4,32
\end{array}
$$


II. Bestimmung. Lösung wie bei obiger Bestimmung bereitet, aber etwa 15 Stunden bei $0^{\circ}$ gestanden.

Das Ergebniss war sehr ähnlich; der Anfangswerth war zwar etwas kleiner, stieg aber ebenso allmählich während der Dauer des Versuches.

$$
\begin{array}{lll}
\mu_{1}=1,96 & \mu_{5}=2,07 & \mu_{9}=2,18 \\
\mu_{2}=1,98 & \mu_{03}=2,09 & \mu_{10}=2,19 \\
\mu_{3}=2,00 & \mu_{7}=2,11 & \mu_{11}=2,22 \\
\mu_{4}=2,02 & \mu_{3}=2,14 & \mu_{13}=2,25
\end{array}
$$

III. Bestimmung. Die bei der I. Bestimmung verwendet gewesene Lösung wurde nach etwa 15 stündigem Stehen wiederum bei $0^{0}$ untersucht.

Die Werthe für $\boldsymbol{\mu}$ waren hier von Anfang an fast um das zehnfache grösser und nahmen dann wieder während der Versuchsdauer zu.

$$
\begin{array}{ll}
\mu_{1}=21,65 & \mu_{3}=23,56 \\
\mu_{3}=21,92 & \mu_{4}=23,68 \\
\mu_{3}=21,94 & \mu_{7}=22,80 \\
\mu_{4}=21,83 & \mu_{S}=23,68
\end{array}
$$

Nach diesen drei Bestimmungen wächst also die Leitfähigkeit der untersalpetrigen Säure während des Durchleitens des Stromes beständig. Es müssen also durch den Einfluss des Wechselstromes Producte aus der untersalpetrigen Säure entstehen, die besser leiten als diese selbst. In der That geben auch derartige Lösungen mit Jodkaliumstärke und Schwefelsäure versetzt sofort eine intensive Blaufärbung; es geht also die bereits oben erwähnte Zersetzung der Säure unter dem Einflusse des Wechselstromes schon bei $0^{0}$ vor sich.

Dass die einmal entstandene salpetrige Säure die Zersetzung der untersalpetrigen Säure in derselben Richtung beschleunigt, geht aus Versuch III hervor, wonach die Leitfähigkeit der bereits einmal dem Wechselstrom ausgesetzten Lösung beim Stehen nach mehreren Stunden fast zehnmal so gross geworden war. Andererseits zeigt Versuch II durch die beträchtliche $\mathrm{Ab}$ nahme der Leitfähigkeit einer bei $0^{0}$ intact gehaltenen Säure- 
lösung, dass die untersalpetrige Säure selbst bei $0^{0}$ langsam zerfällt und zwar ganz überwiegend, wenn nicht ausschliesslich, in Stickoxydul und Wasser.

Als wahrscheinlichster Werth für die molekulare Leitfähigkeit der untersalpetrigen Säure darf wohl der erstbeobachtete angesehen werden; es ist also bei $v=64$

$$
\mu=\sim 3 \text {. }
$$

Die Bestimmung der Leitfähigkeit bei grösseren Verdünnungen und die Berechnung einer Dissociationsconstanten ist natürlich bei einer so schlecht leitenden und zudem so leicht zersetzlichen Verbindung nicht ausführbar.

Jedenfalls ist aber die untersalpetrige Säure entgegen einer weitverbreiteten Meinung keine starke Säure; sie ist im Gegentheil etwa von derselben Grössenordnung wie die Kohlensäure. (Nach $\mathrm{Knox}^{19}$ ) ist die Dissociationsconstante der Kohlensäure $\mathrm{k}=0,00000008$.)

Beide Säuren<smiles>O=C(O)O[W]</smiles>

sind also sehr schwach und darin wieder einander ähnlich.

\section{Leitfähigkeit des Natriumhyponitrites.}

Da sich schon bei den oben erwähnten Titrationsversuchen das saure Natriumsalz $\mathrm{NaHN}_{2} \mathrm{O}_{2}$ als sehr unbeständig gezeigt batte, wurde nur das neutrale Salz auf Leitfähigkeit untersucht. Eine solche Lösung konnte nicht durch Eintragen von aberschüssigem Silberhyponitrit in eine Chlornatriumlösung bereitet werden, da sich beide Salze nur schwierig, d. i. sehr langsam und unvollständig umsetzen, sondern nur durch Zusammengeben äquivalenter Mengen von untersalpetriger Säure und Natronlauge. Die untersalpetrige Säure wurde hierzu in der bekannten Weise aus Silberhyponitrit und Normal-Salzsäure, die Natronlauge nach den Angaben von Hantzsch und Geri-

19) Zeitschr. f. phys. Chem. 17, 185. 
lowski ${ }^{20}$ ) bereitet. Die bei $0^{0}$ vermischten Lösungen wurden durch entsprechendes Verdünnen $1 / 32$ normal gemacht. Sämmtliche Bestimmungen wurden bei $0^{0}$ ausgeführt.

I. Leitfähigkeit für $v=64$. Es wurden sieben aufeinanderfolgende Einstellungen gemacht:

$$
\begin{array}{ll}
\mu_{1}=111,66 & \mu_{5}=110,26 \\
\mu_{2}=110,76 & \mu_{6}=109,26 \\
\mu_{3}=109,56 & \mu_{7}=109,16 \\
\mu_{1}=109,56 &
\end{array}
$$

II. Dasselbe für $v=128$ und sechs direct aufeinanderfolgende Einstellungen:

$$
\begin{array}{ll}
\mu_{1}=139,06 & \mu_{4}=134,42 \\
\mu_{2}=137,06 & \mu_{5}=134,12 \\
\mu_{3}=135,46 & \mu_{65}=132,92
\end{array}
$$

III. Dasselbe für $v=256$ und fünf Einstellungen unter Beobachtung der Zeit:

$$
\begin{array}{rlrl}
\text { Zur Zeit } 0: \mu & =148,04 & \text { nach } 16^{\prime}: \mu=139,64 \\
\text { nach } 7^{\prime}: \mu & =143,04 & \text { nach } 15^{\text {h }}: \mu=126,34 \\
\text { nach } 13^{4}: \mu & =141,14 & &
\end{array}
$$

Bei allen drei Bestimmungsreihen zeigt sich die auffallende Thatsache, dass die Werthe der molekularen Leitfähigkeit von Einstellung zu Einstellung abnehmen. Als Erklärung hierfür können zwei Gründe angenommen werden. Erstens kann durch Anziehen von Kohlensäure aus der Luft die Leitfähigkeit der stark alkalisch reagirenden Lösung des Natriumhyponitrites herabged̉ückt werden. Dass dies wahrscheinlich ist, zeigen die analogen Erfahrungen bei der Leitfähigkeitsbestimmung des Natriumcarbonates. Dann aber kann der Strom selbst schwächer leitende Zersetzungsproducte aus dem stark bydrolysirten Natriumhyponitrit erzeugen, was bei der Bildung von Nitriten ebenfalls der Fall sein müsste.

Trotz dieser störenden Einflüsse ist es deutlich erkennbar, dass die Leitfähigkeit mit steigender Verdünnung sehr bedeutend

29) Ber. d. dentsch. chem. Ges. 29,747 . 
zunimmt. Freilich kann infolge der durch den Strom hervorgerufenen Veränderungen den einzelnen Werthen eine besondere Zuverlässigkeit nicht zuerkannt werden. Als wahrscheinlichster Werth kann wohl nur der erstbeobachtete angesehen werden:

$$
\text { für } v=64 \text { und } \mathrm{t}=0^{0} \text { ist }: \mu=\sim 112 \text {. }
$$

Jedenfalls weist der grosse Werth auf eine sehr weitgehende hydrolytische Spaltung des Dinatriumsalzes hin; welche sich auch in seinem Verhalten gegen Phenolphtaleïn ausprägt.

Vergleich des Nitramides mit der untersalpetrigen Säure.

Derselbe wurde, soweit er nicht bereits aus den Eigenschaften der freien untersalpetrigen Säure hergeleitet werden kann, durch die Bestimmungen des Molekulargewichtes und der Leitfähigkeit des Nitramids vervollständigt.

Die Molekulargewichtsbestimmung des Nitramids, welche anscheinend noch nicht ausgeführt worden ist, ergab zufolge eines von Herrn M. K alb angestellten Versuches in wässriger Lösung dieselbe Grösse, die wir für die untersalpetrige Säure gefunden haben. Schon hiernach kann das Nitramid nur sehr wenig dissociirt sein.

$$
\begin{gathered}
\text { Berechnet für } \\
\mathrm{H}_{2} \mathrm{~N}_{2} \mathrm{O}_{2}
\end{gathered}
$$$$
62
$$

Gefunden

Leitfähigkeitsbestimmung des Nitramides.

I. Bestimmung. $v=32$; Temperatur: $0^{\circ}$.

Es wurden fünf aufeinanderfolgende Versuchsreihen, jede zu vier Einstellungen gemacht und aus diesen der Mittelwerth jeder Versuchsreihe berechnet.

3. Versuchsreihe : $\mu=1,97$

4. Versuchsreihe: $\mu=1,98$

2. $\quad, \quad \mu=1,95$

5. $\quad, \quad \mu=1,96$

3. $\quad " \quad \mu=1,93$

II. Bestimmung. $v=64$; Temperatur: $0^{0}$.

Es wurden zwei aufeinanderfolgende Versuchsreihen, jede zu drei Einstellungen ausgeführt.

1. Versuchsreihe : $\mu=1,69$;

2. Versuchsreihe: $\mu=1,69$. 
340 Hantzsch, Zur Isomerie der Verbindungen $\mathrm{N}_{2} \mathrm{O}_{2} \mathrm{H}_{2}$.

Diesen Bestimmungen zufolge ist das Nitramid ebenso wenig wie die untersalpetrige Säure eine starke Säure, wie Thiele ${ }^{21}$ ) meint, sondern eine sehr schwache Säure. Das geringe Leitvermögen macht auch hier die Bestimmung einer Dissociationsconstanten unmöglich. Immerhin sind die Werthe für Nitramid ( $u \sim 2$ ) kleiner als die für untersalpetrige Säure $(\mu \sim 3)$. Letztere ist also etwas stärker als ersteres. Im Unterschiede zur untersalpetrigen Säure bleibt aber die. Leitfähigkeit beim Nitramid unter dem Einflusse des Wechselstromes constant. Nitramid ist also auch hiernach im freien Zustande erheblich beständiger als untersalpetrige Säure.

Umlagerungsversuche waren bei der Isomerie der zwei Verbindungen $\mathrm{H}_{2} \mathrm{~N}_{2} \mathrm{O}_{2}$ zumal bei ihrer in vieler Hinsicht doch bedeutenden Aehnlichkeit (Löslichkeitsverhältnisse, Stärke u.s. w.) wenigstens angezeigt. Zahlreiche zu diesem Zweck angestellte Versuche - untersalpetrige Säure wurde aus Benzol, Chloroform, Acetylchlorid umkrystallisirt; Nitramid wurde in ätherischer Lösung mit Jod, Salzsäure, Ammoniak behandelt - führten jedoch bisher nicht von der einen zur anderen Verbindung.

Die hier ermittelten Thatsachen worden in der folgenden Abhandlung zur Begründung einer neuen Auffassung der Isomerie zwischen untersalpetriger Säure und Nitramid herangezogen werden.

\section{Zur Isomerie der Verbindungen $\mathrm{N}_{2} \mathrm{O}_{2} \mathrm{H}_{2}$; von $A$. Hantzsch.}

Die rorausgehende Untersuchung über die untersalpetrige Säure und über ihren Vergleich mit dem ihr isomeren sogenannten Nitramid ist durch dieselben allgemeineren theoretischen Anschauungen hervorgerufen worden, welche mich nach Entdeckung der zwei isomeren Diazometallsalze $\mathrm{R}_{2} \mathrm{~N}_{2} \mathrm{OMe}$ zur Begründung der Stereochemie der Diazokörper geführt haben. Dieselben

21) Diese Annalen $\mathbf{2 8 8 ,} 267$. 